\title{
The dynein-triggered ciliary motion in embryonic nodes: An exploratory study based on computational models
}

\author{
Duanduan Chen $^{\mathrm{a},{ }^{*}}$, Yi Zhong ${ }^{\mathrm{a}}$, Kyosuke Shinohara ${ }^{\mathrm{b}}$, Tomoki Nishida ${ }^{\mathrm{c}}$, Toshiaki Hasegawa ${ }^{\mathrm{c}}$ \\ and Hiroshi Hamada ${ }^{\mathrm{b}}$ \\ ${ }^{a}$ School of Life Science, Beijing Institute of Technology, Beijing, China \\ ${ }^{b}$ Developmental Genetics Group, Graduate School of Frontier Biosciences, Osaka University, Osaka, \\ Japan \\ ${ }^{c}$ Research Center for Ultra-high Voltage Electron Microscopy, Osaka University, Osaka, Japan
}

\begin{abstract}
The cilia, presenting a rotational movement in the embryonic nodes, play a crucial role in the left-right specification during embryogenesis. The characteristic architecture of these cilia is based on a cylindrical arrangement of 9 doublet microtubules and the motion of the cilia is triggered by the dynein motors located between adjacent doublets by converting the chemical energy into mechanical work. Restricted by the inherent difficulties of experiments, the dynein activation patterns in moving cilia cannot be directly observed. Thus, the mechanism of nodal ciliary movement is still unclear. In this study, we present computational models of the nodal ciliary ultrastructure based on tomographic images of the ciliary body. By employing time accurate three-dimensional solid mechanics analysis, we investigate the dynein-triggered sliding between adjacent doublet microtubules and simulate the induced ciliary bending. As an exploratory study, two dynein activation patterns are proposed and their rationality is discussed. The mathematical model presented by this paper provides a platform to investigate various assumptions of dynein activity, facilitating us to propose the most possible dynein activation pattern and therefore improving our understandings regarding the protein-beating problems of cilia.
\end{abstract}

Keywords: Cilia, dynein, ultrastructure, embryonic node, finite element analysis

\section{Introduction}

The left-right specification during embryogenesis is triggered by a leftward flow across a transient embryo structure, the node [1-6]. There are hundreds of mono-cilia located in the embryonic node and the leftward nodal flow is produced by the rotational movement of these cilia [7]. Effective movement of nodal cilia plays a crucial role in correctly directing left-right specification; however, the mechanism that produces nodal ciliary motion is still unclear.

The ultrastructure of embryonic cilia contains a cylindrical arrangement of 9 doublet microtubules. These hollow fibres are made by spontaneous self-assembly and present dynamic dissipative character.

\footnotetext{
${ }^{*}$ Corresponding author: Duanduan Chen, School of Life Science, Beijing Institute of Technology, Beijing, China. Tel.: +86 10 68912154; Fax: +86 10 68915956; E-mail: duanduan@bit.edu.cn.
} 


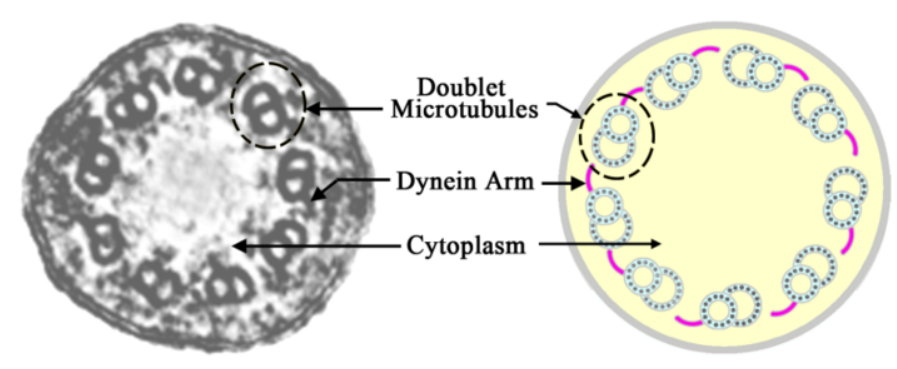

Fig. 1. The ultrastructure of embryonic cilia.

The microtubules are the main elastic elements in the axoneme; however, due to the difficulties in experiments in vivo, their mechanical properties have not been precisely revealed. Recent studies regarding the vibrations indicate viscous damping in microtubules is large and cannot produce resonant vibrations [8]. As shown in Figure 1, dynein arms present between adjacent doublets. By converting the chemical energy of ATP hydrolysis into mechanical work, these motor proteins induce doublet sliding [9], and together with other passive components, such as the mediated cytoplasm, in the cilia, they produce the ciliary motility. Current observation techniques can hardly capture the dynein activity in moving cilia, restricting studies of the mechanism of ciliary motion and thus encouraging researchers to solve the problem from the computational modelling aspect.

In this paper, we present $3 \mathrm{D}$ computational models of the ciliary ultrastructure based on laboratory observed images. By applying dynein forces between adjacent doublets, we simulate the proteintriggered deformation of the cilium induced by linear and sine dynein 'walking' patterns. By comparing the simulated ciliary motion to our observed data, we evaluate the rationality of these dynein activation patterns and discuss the mechanism of nodal ciliary motion.

\section{Methods}

\subsection{The protein-structure model}

According to experimental observations [10], the ciliary body is modelled as a composite of a cylindrical body $(2 \mu \mathrm{m}$ in height, $0.2 \mu \mathrm{m}$ in diameter $)$ with a parabolic tip $(0.2 \mu \mathrm{m}$ in height). The doublet microtubules consist of a complete A-tubule and an incomplete B-tubule. The cross-section of the doublet microtubule is thus modelled as an ellipse with a diameter of $0.03 \mu \mathrm{m}$ in the major axis and $0.17 \mu \mathrm{m}$ in the minor axis. Figure 2(a) displays this model. The spatial resolution of the cilium involves 76 points in the vertical direction of the cylindrical body. This indicates a $24 \mathrm{~nm}$ distance between the grid nodes in the longitudinal direction, corresponding to the actual dynein intervals along the microtubules as observed in our experiments. The tip of the ciliary model is discretized using an unstructured tetrahedral grid and the cylindrical body including the doublet microtubules are extruded by triangular prisms. The grid configuration of the model consists of a total of 111,815 cells.

\subsection{Establishment of the realistic model}

The realistic model of the nodal cilium is established based on the tomographic images of the embryonic node, obtained by ultra-high voltage electron microscopic tomography (UHVEMT). $1 \mu \mathrm{m}$ 
(a)

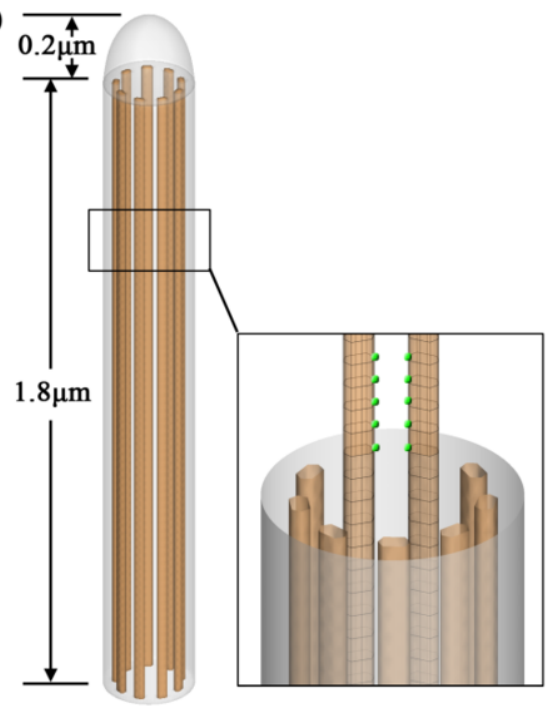

(b)

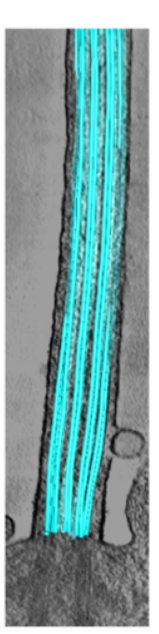

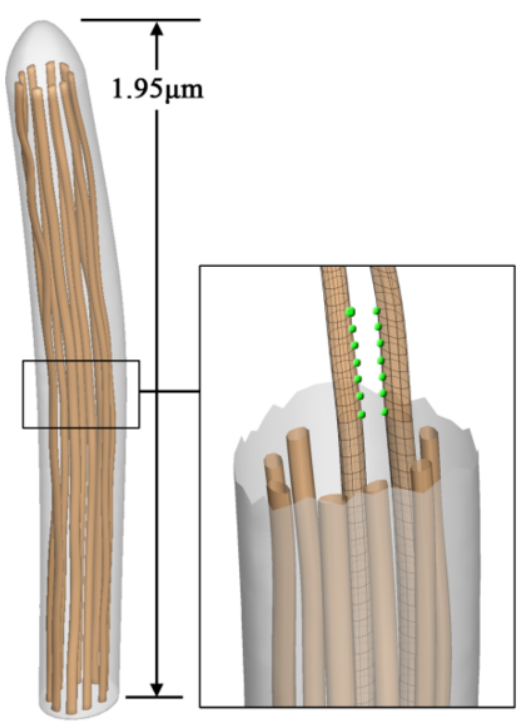

Fig. 2. The computational models. (a) displays the artificial model and (b) shows the realistic model. The enlarged images in (a) and (b) show the grid discretization in two doublets and the green points indicate the dynein force application sites.

thick sections were cut and mounted on molybdenum grids. The sections were observed in an ultrahigh voltage electron microscope operating at $2 \mathrm{MeV}(\mathrm{H}-3000$, Hitachi) and the images were taken at $20,000 \times, \pm 60^{\circ}$, at $2^{\circ}$ intervals, and recorded with a $4096 \times 4096$ F415S slow scan charge-coupled device camera with a pixel size of $15 \mu \mathrm{m}$ (TVIPS, Germany). 3D reconstruction was performed using the IMOD image-processing package. As shown in Figure 2(b), the spatial positions of the microtubules and ciliary membrane were extracted in the tomographic image of the nodal cilium and were exported to CFD-GEOM (ESI Group, France) to establish the 3D model. The distance between adjacent grid nodes along double microtubules was again assigned to $24 \mathrm{~nm}$ and total grid resolution of the model contains 118,540 cells.

\subsection{Mechanical properties}

The elastic modulus of doublet microtubule is reported ranging between $0.05 \sim 5 \mathrm{GPa}$ [11-13]. In this study, we assume the doublet microtubules are homogeneous and isotropic material and the elastic modulus is $0.1 \mathrm{GPa}$. Literatures about the elastic properties of ciliary cytoplasm are lacking. Relative studies indicate that the elastic modulus of cytoplasm may range between $25 \mathrm{~Pa} \sim 5 \times 10^{5} \mathrm{~Pa}[14,15]$. In the current study, the mediated cytoplasm is modelled as solid. A parameter study has been conducted and the elastic modulus of the modelled cytoplasm is finally assigned to be $50 \mathrm{~Pa}$. This value allows the most flexibility of ciliary movement and is close to the limit of what can be realistically computed in terms of local mesh deformations for the ultrastructure of the cilium.

\subsection{Scenarios of the dynein activation}

When activated, each dynein motor located on one doublet microtubule forms a transient attachment to its adjacent doublet and pushes it tipwards [16]. This mechanical function of the dynein allows us to model them as pairs of point loads working in opposite directions along the doublets. As shown in 
Figure 2, the grid discretization is with a $24 \mathrm{~nm}$-interval along the doublet, which is just the spatial interval of dyneins as observed, and therefore, the point loads which mimic the dynein force can be added at the grid points in the model. The force exerted by a single dynein arm is reported varying from 1 to $10 \mathrm{pN}$ [17-21]. Here, we assume the force generated by a single dynein arm is $5 \mathrm{pN}$. The initiation of dynein activity moves sequentially from doublet to doublet in about 1/9 cycle [22]; the dynein activity along one doublet occurs once per cycle. The rotational cycle of nodal cilia is $10 \mathrm{~Hz}$ [23]. The protein motors along one pair of adjacent doublets, therefore, function effectively in $0.011 \mathrm{~s}$.

Previous literatures indicate the magnitude, direction, and operation time of dynein force in cilia. However, the sequential manner of dynein activation is still unclear. Our previous study indicates that the dynein attachment could be a distance-controlled process $[24,25]$ and the dynein activation between pairs of doublets is transferred clockwise. In other words, when the dynein bridges finish establishment between doublet $\mathrm{N}$ and $\mathrm{N}+1$ (the doublets are numbered clockwise), in the next 1/9 cycle, the dyneins between doublet $\mathrm{N}+1$ and $\mathrm{N}+2$ are activated, while, the dynein bridges between doublet $\mathrm{N}$ and $\mathrm{N}+1$ are detaching. This previous work provides the dynein activation sequence among the 9 doublets; however, to produce a circular rotation of the cilia, we still need to clarify the dynein activation pattern between one pair of doublets, i.e., how the dynein protein 'walks' along the microtubule.

In the current work, since the base cell supplies energy to the protein motors, we assume the dynein bridges 'walk' from the base to the tip. More specifically, we investigate two types of dynein activation pattern: linear and sine. We firstly test the dynein activation scenarios on the artificial model, simulate the induced ciliary motion, and by comparing to observations, the rationality of the scenarios can be evaluated and the possible dynein 'walking' pattern can be proposed. Then, the selected dynein activation pattern is applied in the realistic model. More realistic ciliary motion can be then simulated. The time-variant climbing height of the dynein bridges on one doublet can be represented by $h=$ $163.64 t \mu \mathrm{m}$ in the linear scenario and $h=1.8 \operatorname{Sin}(142.73 t) \mu \mathrm{m}$ in the sine scenario; and the detaching height of dyneins in the next $1 / 9$ cycle is $h=1.8-163.64 t \mu \mathrm{m}$ in the linear scenario and $h=1.8-1.8 \mathrm{Sin}$ $(142.73 t) \mu \mathrm{m}$ in the sine scenario.

\subsection{Numerical algorithms}

The motility of cilia is calculated by embedding the produced forces on the discretized elements (properly located in space and time according to the scenarios we discussed in the previous section). The problem is solved by using a stress solver in CFD-ACE+ (ESI CFD, France), based on the Finite Element method. The stress solver allows explicit input of the point loads on the grid nodes, which facilitates us simulating the transient dynein forces in the model. Moreover, the computational platform we used allows the concurrent embedding of the forces-generating elements of the axoneme, along with the passive deformable elements of the structure into a unified framework, permitting the computation of self-induced deformation and motion for this cilium system. The Solid-body Elasticity Analogy method [26] is employed in this computational work to calculate and update mesh deformation, where the re-meshing problem is solved by calculating the displacement between the moved boundaries and based on the solution of the equations of linear elasticity.

\section{Results and discussion}

In both of the artificial and realistic models, the computation has been implemented for 3 cycles and the ciliary motion presented below is exported based on the final cycle. 

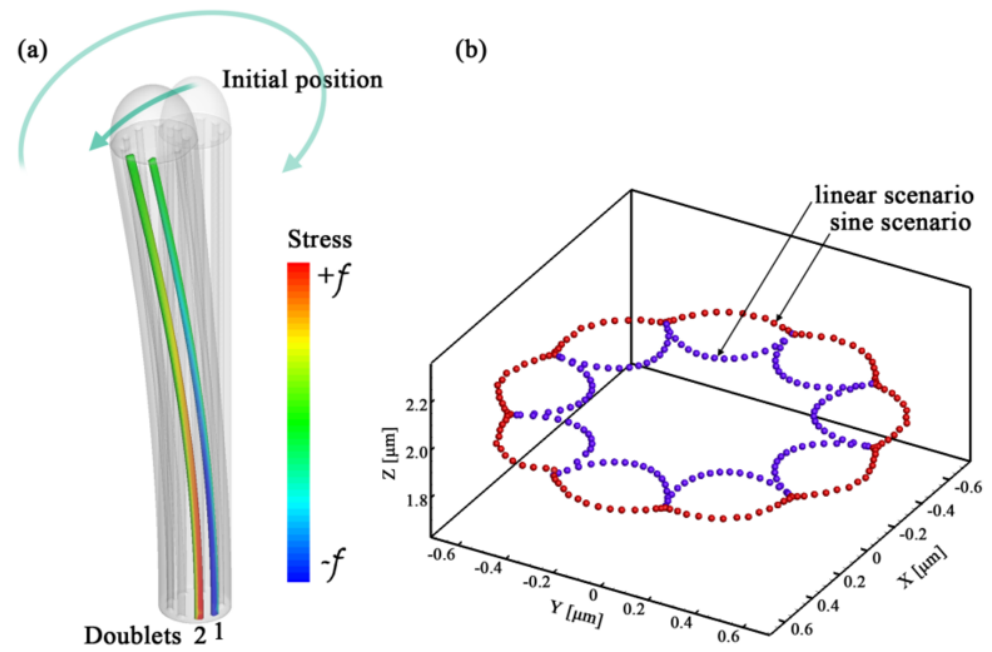

Fig. 3. The computational results of the artificial model. (a) displays the dynein-triggered ciliary bending, and (b) shows the resulting tip trajectory of the cilium.

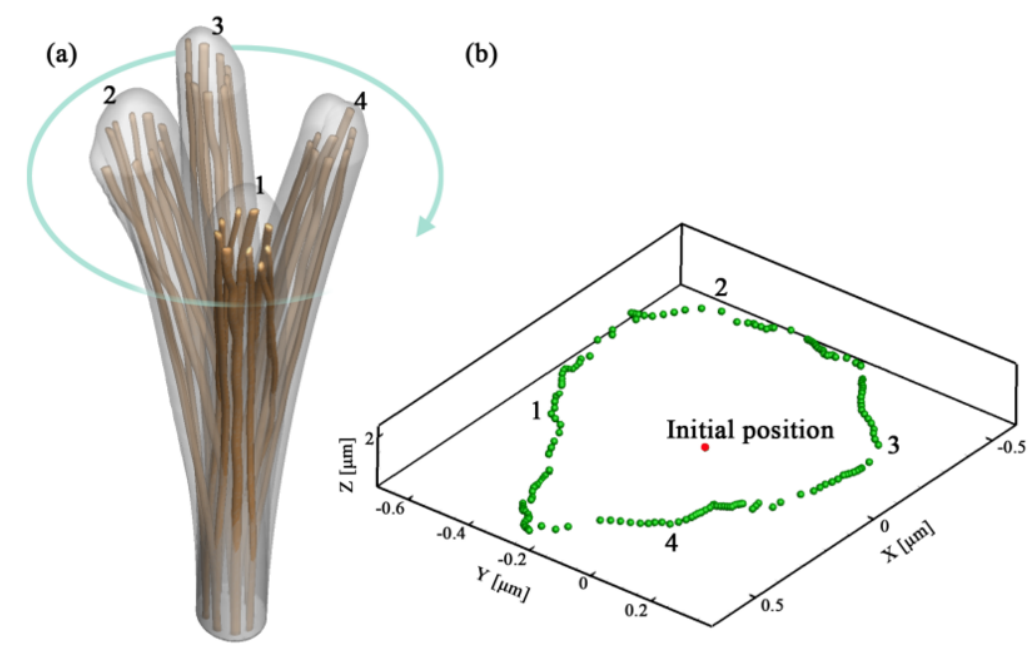

Fig. 4. The results of the realistic model. (a) displays ciliary rotational movement, and (b) shows the tip trajectory.

Figure 3(a) shows the dynein climbing up from the base to the tip between doublet 1 and 2. The sliding forces generated by them induce the accumulation of internal forces and it is exactly those forces that produce the structural bending towards the higher-numbered doublet direction (doublet 2, or generally doublet $\mathrm{N}+1$ ). At the end of $1 / 9$ cycle, the ciliary bending reaches the maximum, which is $0.46 \mu \mathrm{m}$ at the ciliary tip. In the next $1 / 9$ cycle, the dynein activation transfers to the next pair of doublets (doublet 2 and 3 ) and the dynein bridges between doublet 1 and 2 begins to detach. This process makes the ciliary movement change from bending to rotating.

Figure 3(b) displays the ciliary tip trajectory of the linear and sine scenarios. Both the dynein "walking' patterns is able to produce the rotational movement of the cilium. However, the actual moving 
trajectory is different; the sine model shows obviously smoother rotation of the cilium than the movement produced by the linear scenario and is similar to experimental observations. The sine model is then applied in the realistic model for further investigation.

Figure 4 displays the motion of the cilium in the realistic model, stimulated by the sine dynein 'walking' pattern. The numbered structures (1-4) in Figure 4(a) display the ciliary configurations at time $0.027 \mathrm{~s}, 0.049 \mathrm{~s}, 0.071 \mathrm{~s}$ and $0.093 \mathrm{~s}$ in one cycle. Figure $4(\mathrm{~b})$ shows the tip trajectory of the cilium. The trajectory points are not evenly distributed as those shown in Figure 3(b). This is because the ciliary ultrastructure is reconstructed based on real ciliary tomographic image; the microtubules are with natural curvature and morphology rather than the straight cylindrical doublets in the artificial model. This indicates the geometry of the doublets in real cilia also plays an role in controlling ciliary motion, implicating that mutant cilia with abnormal microtubules may present different types of motion. The maximum deformation of the cilium occurs at $0.071 \mathrm{~s}$ in one cycle, which is $0.53 \mu \mathrm{m}$ at the ciliary tip.

A few studies have been conducted to reveal the mechanism of ciliary motility, such as the curvature-controlled model proposed by Brokaw [27], the geometric clutch model by Lindemann [28], and the switch-point model by Satir [29]. More recently, Dillon et al. [30] established a 2D model to simulate the axoneme that consists of one pair of microtubules embedded in a viscous incompressible fluid. Mitran [31] modelled the microtubule structures by large-deflection, curved, finite-element beams. These studies brought important insight into the mechanism of dynein activation, however, most of them are limited by dimensions and focus on " $9+2$ " cilia. The current study we proposed is able to represent the realistic ciliary ultrastructure in $3 \mathrm{D}$ and embed the dynein forces explicitly. The deformation of the cilium is relatively small comparing to laboratory observations (probably due to the underestimation of dynein forces) yet coherent and comparable in shape. Further improvement of this model and more precise simulations would be pursued by improving the physical parameters of the cilium when relative information from biological studies becomes available. Nevertheless, this proteinstructure model sheds light on the dynein-microtubule motor mechanism of cilia and proposes a possible dynein activation pattern for the nodal cilia. It also enables future experimental studies on the influence of specific axoneme defects.

\section{Conclusion}

In this study, we establish two computational models of the ciliary ultrastructure; the artificial model is designed based on the ciliary geometric information, while the realistic model is reconstructed from the real tomographic images of nodal cilia. Linear and sine dynein 'walking' patterns have been applied in the artificial model firstly. The difference of the induced trajectories is discussed and the results indicate the sine pattern of dynein activation can produce better ciliary movement results when comparing to experimental observations. The sine pattern is then applied in the realistic model and the ciliary motion is simulated. It results in a rotational movement of the cilium as observed in experiments, confirming the sine pattern dynein 'walking' and detaching process is a possible dynein activation sequence in embryonic cilia.

\section{Acknowledgement}

This study is supported by the National Natural Science Foundation of China (31200704). We would like to acknowledge the ESI Group and Mr. Xiangde Zhu for allowing us to use CFD-ACE+. 


\section{References}

[1] S. Takeda et al., Left-right asymmetry and kinesin superfamily protein KIF3A: New insights in determination of laterality and mesoderm induction by kif3A(-/-) mice analysis, J. Cell. Biol. 145 (1999), 825-836.

[2] S. Nonaka et al., Randomization of left-right asymmetry due to loss of nodal cilia generating leftward flow of extraembryonic fluid in mice lacking KIF3B motor protein, Cell 95 (1998), 829-837.

[3] Y. Okada et al., Abnormal nodal flow precedes situs inversus in iv and inv mice, Mol. Cell 4 (1999), 459-468.

[4] J.J. Essner et al., Conserved function for embryonic nodal cilia, Nature 418 (2002), 37-38.

[5] H. Hamada et al., Establishment of vertebrate left-right asymmetry, Nat. Rev. Genet. 3 (2002), 103-113.

[6] J.R. Marszalek et al., Situs inversus and embryonic ciliary morphogenesis defects in mouse mutants lacking the KIF3A subunit of kinesin-II, Proc. Natl. Acad. Sci. 96 (1999), 5043-5048.

[7] J.H. Cartwright, O. Piro and I. Tuval, Fluid-dynamical basis of the embryonic development of left-right asymmetry in vertebrates, Proc. Natl. Acad. Sci. 101 (2004), 7234-7239.

[8] K.R. Foster and J.W. Baish, Viscous damping of vibrations in microtubules, J. Biol. Phys. 26 (2000), 255-260.

[9] S.A. Burgess et al., Dynein structure and power stroke, Nature 421 (2003), 715-718.

[10] S. Nonaka et al., De novo formation of left-right asymmetry by posterior tilt of nodal cilia, PLoS Biol. 3 (2005), e268.

[11] P. Venier, A.C. Maggs, M.F. Carlier and D. Pantaloni, Analysis of microtubule rigidity using hydrodynamic flow and thermal fluctuations, J. Biol. Chem. 269 (1994), 13353-13360.

[12] S.A. Baba, Flexural rigidity and elastic constant of cilia, J. Exp. Biol. 56 (1972), 459-467.

[13] D.R. Peterson, Biomechanics, in: The Biomedical Engineering Handbook, J.D. Bronzino, ed., CRC Press, Florida, 2006, pp. 47-66.

[14] V.A. Parsegian, N. Fuller and R.P. Rand, Measured work of deformation and repulsion of lecithin bilayers, Proc. Natl. Acad. Sci. 76 (1979), 2750-2754.

[15] F.J. Byfield et al., Cholesterol depletion increases membrane stiffness of aortic endothelial cells, Biophys. J. 87 (2004), 3336-3343.

[16] L.A. Fox and W.S. Sale, Direction of force generated by the inner row of dynein arms on flagellar microtubules, J. Cell. Biol. 105 (1987), 1781-1787.

[17] I. Minoura, T. Yagi and R. Kamiya, Direct measurement of inter-doublet elasticity in flagellar axonemes, Cell Struct. Funct. 24 (1999), 27-33.

[18] C. Shingyoji, H. Higuchi, M. Yoshimura, E. Katayama and T. Yanagida, Dynein arms are oscillating force generators, Nature 393 (1998), 711-714.

[19] C.B. Lindemann, Structural-functional relationships of the dynein, spokes, and central-pair projections predicted from an analysis of the forces acting within a flagellum, Biophys. J. 84 (2003), 4115-4126.

[20] K.A. Schmitz, D.L. Holcomb-Wygle, D.J. Oberski and C.B. Lindemann, Measurement of the force produced by an intact bull sperm flagellum in isometric arrest and estimation of the dynein stall force, Biophy. J. 79 (2000), 468-478.

[21] Z. Teff, Z. Priel and L.A. Ghebery, Forces applied by cilia measured on explants from mucociliary tissue, Biophys. J. 92 (2007), 1813-1823.

[22] R.N. Seetharam and P. Satir, Coordination of outer arm dynein activity along axonemal doublet microtubules, Cell Motil Cytoskeleton 65 (2008), 572-580.

[23] J.H. Cartwright et al., Embryonic nodal flow and the dynamics of nodal vesicular parcels, J. R. Soc. Interface 4 (2007), 49-55,

[24] D. Chen, D. Norris and Y. Ventikos, Ciliary behaviour and mechano-transduction in the embryonic node: Computational testing of hypotheses, Med. Eng. Phys. 33 (2011), 857-867.

[25] D. Chen, D. Norris and Y. Ventikos, Chemosignalling, mechanotransduction and ciliary behaviour in the embryonic node: Computational evaluation of competing theories, Proc. Inst. Mech. Eng. H 228 (2014), 465-476.

[26] A.A. Johnson and T.E. Tezduyar, Mesh update strategies in parallel finite-element computations of flow problems with moving boundaries and interfaces, Comput. Methods in Appl. Mech. Eng. 119 (1994), 73-94.

[27] C.J. Brokaw, Computer simulation of flagellar movement, IX. oscillation and symmetry breaking in a model for short flagella and nodal cilia, Cell Motil Cytoskeleton 60 (2005), 35-47.

[28] C.B. Lindemann, Experimental evidence for the geometric clutch hypothesis, Curr. Top Dev. Biol. 95 (2011), 1-31.

[29] P. Satir and T. Matsuoka, Splitting the ciliary axoneme: Implications for a "switch-point" model of dynein arm activity in ciliary motion, Cell Motil Cytoskeleton 14 (1989), 345-58.

[30] R.H. Dillon, L.J. Fauci and C. Omoto, Mathematical modeling of axoneme mechanics and fluid dynamics in ciliary and sperm motility, Dynamics of Continuous Discrete and Impulsive Systems-Series A-Mathematical Analysis 10 (2003), 745-757.

[31] S.M. Mitran, Metachronal wave formation in a model of pulmonary cilia, Comp. Struct. 85 (2007), 763-774. 